\title{
Temperature Dependence of Optical Linewidths and Specific Heat of Rare-Earth-Doped Silicate Glasses
}

\author{
Th. Schmidt, ${ }^{1}$ J. Baak, ${ }^{2}$ D. A. van de Straat ${ }^{2}$ H. B. Brom,${ }^{2}$ and S. Völker ${ }^{1, *}$ \\ ${ }^{1}$ Center for the Study of Excited States of Molecules, Huygens and Gorlaeus Laboratories, Leiden University, \\ P.O. Box 9504, 2300 RA Leiden, The Netherlands \\ ${ }^{2}$ Kamerlingh Onnes Laboratory, Leiden University, P.O. Box 9506, 2300 RA Leiden, The Netherlands
}

(Received 9 April 1993)

\begin{abstract}
Optical dephasing and specific heat measurements were performed on two rare-earth-doped silicate glasses. The effective homogeneous linewidth of the optical transition, $\Gamma_{\text {hom }}$, was compared to the two-level-system (TLS) contribution to the specific heat, $C_{v}^{\mathrm{TLS}}$, between 0.4 and $12 \mathrm{~K}$. Both $\Gamma_{\text {hom }}$ and $C_{v}^{\text {TLS }}$ vary as $T^{\alpha}$ with the same value of $\alpha$ for a given rare-earth/glass sample, but with different $\alpha$ for the two samples ( $\alpha=1.3$ for the $\mathrm{Pr}^{3+} /$ glass, $\alpha=1.0$ for $\mathrm{Eu}^{3+} /$ glass). From the ratio of $\Gamma_{\text {hom }}$ to $C_{v}^{\text {TLS }}$ for each glass, it appears that the coupling strengths to the TLS for each rare-earth optical transition are similar, but that the density of TLS differ due to their different compositions.
\end{abstract}

PACS numbers: 78.50.Ec, 33.70.Jg, 61.43.Fs, 65.40.Em

Low-temperature properties of glasses at $T<5 \mathrm{~K}$ have been extensively studied by experiment and theory during the last two decades and shown to be different from crystals [1]. For example, the specific heat of glasses increases almost linearly with temperature, and the thermal conductivity quadratically [1-3]. The optical properties of chromophores in glasses at low temperature also are different from those in crystals: The inhomogeneous linewidths are very broad and the homogeneous widths have a weak temperature dependence, between $T^{1.0}$ and $T^{2.0}$ for $T<30 \mathrm{~K}[4-7]$.

Very-low-frequency modes with energies smaller than $0.1 \mathrm{meV}$, called two-level or tunneling systems (TLS), are assumed to be responsible for the low-temperature thermal, acoustic, and dielectric properties of glasses. Although the origin of these low-frequency vibrations is still not known and presently much debated [8], the TLS are supposed to consist of a distribution of double-well potentials in which atoms or groups of atoms can tunnel from one potential minimum to another with an almost constant density of states [9].

The broad distribution of parameters associated with the TLS gives rise to a broad distribution of rates of dynamical processes. Structural changes in the glass, for example, which can also be modeled as tunneling between these double wells, lead to spectral diffusion of the optical homogeneous linewidth. As a consequence, the measured or "effective" homogeneous linewidth, $\Gamma_{\text {hom }}$, will depend on the characteristic time scale $\left(\tau_{\exp }\right)$ of the experiment [1]. Differences observed in the value of $\Gamma_{\text {hom }}$ obtained by two techniques for the same system, like two-pulse photon echoes $\left(\tau_{\text {exp }} \simeq 100\right.$ ps to 100 $\mathrm{ns}$ ) and hole burning $\left(\tau_{\exp } \simeq 100 \mathrm{~s}\right.$ ) for $\mathrm{Nd}^{3+}$ in fused silica glass $[10,11]$ and for various organic glassy systems $[12,13]$, and two-pulse versus stimulated three-pulse echoes $[14,15]$ have been attributed to spectral diffusion (SD). Also results from hole-burning experiments measured on different time scales were reported to be due to the same effect [16-18]. The temperature dependence of $\Gamma_{\text {hom }}$ (for $T<5 \mathrm{~K}$ ), however, has been found to be independent of the time scale of the experiment, within the measured accuracy $[11,13,18]$.

Many models based on the interaction of TLS with the optical transition have been proposed to explain the optical line broadening of chromophores doped in glasses [10,14,19-22]. They predict that both the homogeneous linewidth and the specific heat should increase with temperature as $T^{\alpha}$ with an exponent $\alpha$ which is expected to be the same for both properties if a dipolar coupling of the optical transition to the TLS is assumed.

Although extensive data on optical dephasing in doped glasses and on the specific heat in undoped glasses have been reported, no comparative measurements of the two properties have been performed on identical samples. We present here results on both the effective homogeneous linewidth and the specific heat as a function of temperature between 0.4 and $12 \mathrm{~K}$ for two rare-earth-doped silicate glasses: an $\mathrm{Eu}^{3+}$ sample with composition (in mol\%) $74.75 \% \mathrm{SiO}_{2}, 15 \% \mathrm{Na}_{2} \mathrm{O}, 5 \% \mathrm{BaO}, 5 \% \mathrm{ZnO}$, and $0.25 \%$ $\mathrm{Eu}_{2} \mathrm{O}_{3}$, and a $\mathrm{Pr}^{3+}$ sample with $59.9 \mathrm{~mol} \% \mathrm{SiO}_{2}, 27.5 \%$ $\mathrm{Li}_{2} \mathrm{O}, 10 \% \mathrm{CaO}, 2.5 \% \mathrm{Al}_{2} \mathrm{O}_{3}$, and $0.1 \% \operatorname{Pr}_{2} \mathrm{O}_{3}$. We have found that the temperature dependence of the effective homogeneous linewidth, $\Gamma_{\text {hom }}$, and of the TLS contribution to the specific heat, $C_{v}^{\text {TLS }}$, are the same for a given system, but different for the two systems.

The width $\Gamma_{\text {hom }}$ of the optical transition ${ }^{1} D_{2} \leftarrow{ }^{3} H_{4}$ of $\mathrm{Pr}^{3+}$ at $606 \mathrm{~nm}$ and that of the ${ }^{5} D_{0} \leftarrow{ }^{7} F_{0}$ transition of $\mathrm{Eu}^{3+}$ at $580 \mathrm{~nm}$ have been determined by spectral hole burning using an amplitude stabilized cw singlefrequency dye laser (bandwidth $\sim 2 \mathrm{MHz}$ ) [23]. The holes were burned with fluence densities, $P_{b} t_{b} / A$, between $1 \mathrm{~mJ} / \mathrm{cm}^{2}$ and $10^{3} \mathrm{~J} / \mathrm{cm}^{2}$, where $P_{b}$ is the burning power of the laser, $t_{b}$ the burning time, and $A$ the area of the laser spot on the sample. The holes were subsequently monitored by fluorescence excitation spectroscopy with the laser intensity reduced by a factor of 
100 and in a time after burning of about $50 \mathrm{~s}$. We define here the value of $\Gamma_{\text {hom }}$ as that obtained by extrapolating the hole half-width, $\frac{1}{2} \Gamma_{\text {hole }}$, to zero burning fluence density, $P_{b} t_{b} / A \rightarrow 0$, and deconvoluting the laser bandwidth. Since spectral diffusion may contribute to the hole width, the homogeneous linewidth that would be obtained on a much shorter time scale could be smaller than $\frac{1}{2} \Gamma_{\text {hole }}$. The temperature dependence of $\Gamma_{\text {hom }}$ for both samples, however, was found to be the same on time scales between $10^{-4}$ and $10^{2} \mathrm{~s}[23,24]$. Also the values of $\Gamma_{\text {hom }}$ were equal on these time scales, which suggests that spectral diffusion may only play a role for $\tau_{\exp }<10^{-4} \mathrm{~s}$. The hole shapes were best fitted with a Lorentzian profile $[7,23]$, which is an indication that the coupling between the optical transition and the TLS is through a dipole-dipole interaction $[10-12,14]$.

The optical experiments were performed between 0.4 and $12 \mathrm{~K}$ for the $\mathrm{Pr}^{3+}$ sample, and between 0.4 and 4.2 $\mathrm{K}$ for the $\mathrm{Eu}^{3+}$ sample. A ${ }^{4} \mathrm{He}$-flow cryostat was used for $T>4.2 \mathrm{~K}$; whereas a ${ }^{4} \mathrm{He}$-bath cryostat was used between 1.2 and $4.2 \mathrm{~K}$, the temperature of which was controlled by means of the vapor pressure of the ${ }^{4} \mathrm{He}$. For $T<1.2 \mathrm{~K} \mathrm{a}{ }^{3} \mathrm{He}$ insert was placed into the ${ }^{4} \mathrm{He}-\mathrm{bath}$ cryostat. The accuracy of the temperature determination was \pm 0.01 to $\pm 0.05 \mathrm{~K}$ depending on the temperature range.

The temperature dependence of $\Gamma_{\text {hom }}$ of the ${ }^{1} D_{2}$ $\leftarrow{ }^{3} H_{4}$ transition of $\mathrm{Pr}^{3+}$ is shown in $\log$-log form in Fig. 1 (open circles). The hole-burning mechanism for $T \leq 2.5 \mathrm{~K}$ is due to optical pumping of the $\mathrm{Pr}^{3+}$ ions [23], a process by which the population of the nuclear hyperfine levels in the electronic ground state is redis-

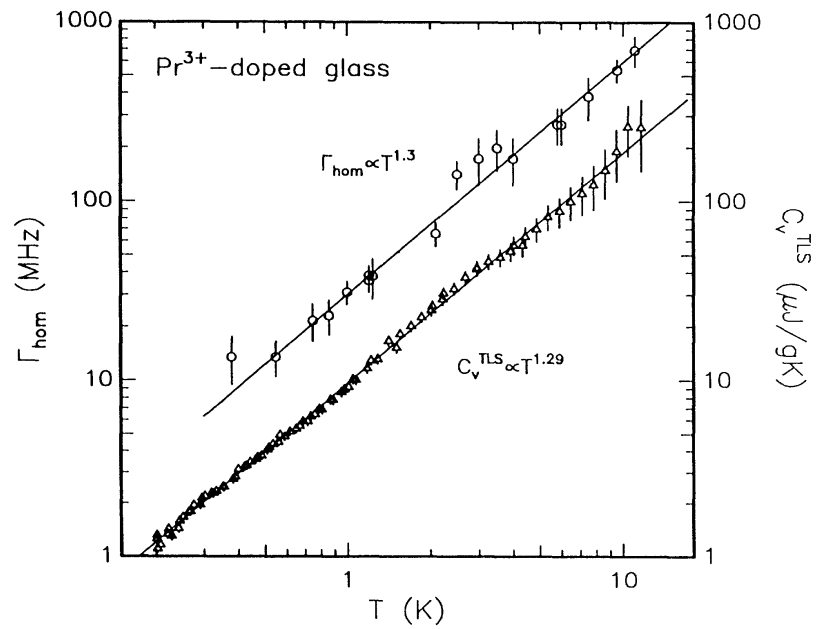

FIG. 1. Homogeneous linewidth, $\Gamma_{\text {hom }}$ (open circles, left scale), and contribution of the two-level systems to the specific heat, $C_{v}^{\mathrm{TLS}}$ (open triangles, right scale), as a function of temperature for the $\mathrm{Pr}^{3+}$-doped silicate glass. The fits yield $\Gamma_{\text {hom }}=(30 \pm 2) T^{(1.3 \pm 0.1)} \mathrm{MHz}$ between 0.4 and $12 \mathrm{~K}$, and $C_{v}^{\mathrm{TLS}}=(9.6 \pm 0.1) T^{(1.29 \pm 0.02)} \mu \mathrm{J} / \mathrm{g} \mathrm{K}$ between 0.1 and $15 \mathrm{~K}$. tributed. For $T \geq 2.5 \mathrm{~K}$ the holes are permanent and due to local rearrangements of the glass surrounding the excited ions $[23,24]$. The best fit to the data is a power law, $\Gamma_{\text {hom }}=a T^{\alpha}=(30 \pm 2) T^{(1.3 \pm 0.1)}$, where $\Gamma_{\text {hom }}$ is given in $\mathrm{MHz}$ (solid line).

A similar log-log plot of $\Gamma_{\text {hom }}$ versus $T$ for the ${ }^{5} D_{0}$ $\leftarrow{ }^{7} F_{0}$ transition of $\mathrm{Eu}^{3+}$ is given in Fig. 2 (solid circles). It was not possible to burn holes at temperatures higher than $4.2 \mathrm{~K}$ because the hole-burning mechanism here is due to optical pumping of nuclear quadrupole levels [24] and above that temperature the spin-lattice relaxation, responsible for refilling of the holes, is faster than the time it takes to probe them. No permanent hole-burning as for $\mathrm{Pr}^{3+}$ has been observed for this system [23]. The best fit to the data of Fig. 2 is again a power law, $\Gamma_{\text {hom }}=$ $a T^{\alpha}=(9 \pm 1) T^{(1.0 \pm 0.1)}$, with $\Gamma_{\text {hom }}$ given in $\mathrm{MHz}$.

The specific heat data were obtained in a dilution refrigerator using a thermal relaxation method [25]. In this method the sample is permanently linked to a heat sink. Averaged temperature profiles are obtained by periodic applications of a heat pulse. The heat capacity follows from the power applied, from the equilibrium temperature reached during the heat pulse, and from the area of the temperature profile in a $T$-versus-time plot [26]. The relaxation times were typically of the order of $10 \mathrm{~s}$. Here the samples of a few tenths of a gram were mounted on top of a sapphire plate of $0.14 \mathrm{~g}$ using a precisely determined amount of Apiezon $\mathrm{N}$ grease (typically a few $\mathrm{mg}$ ). As thermometer a ruthenium-oxide resistor was used. A $\mathrm{NiCr}$ heater was sputtered on the bottom side of the sapphire plate and a thin Au wire provided the connection to the heat sink. All experimental data were corrected

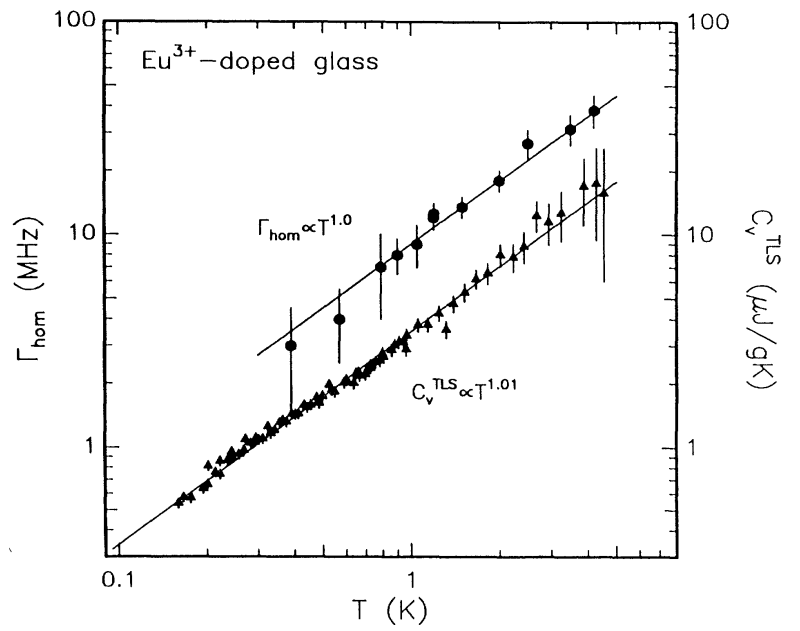

FIG. 2. Homogeneous linewidth, $\Gamma_{\text {hom }}$ (solid circles, left scale), and two-level-system contribution to the specific heat, $C_{v}^{\text {TLS }}$ (solid triangles, right scale), for the $\mathrm{Eu}^{3+}$-doped silicate glass. The fits yield $\Gamma_{\text {hom }}=(9 \pm 1) T^{(1.0 \pm 0.1)} \mathrm{MHz}$ between 0.4 and $4.2 \mathrm{~K}$, and $C_{v}^{\mathrm{TLS}}=(3.5 \pm 0.1) T^{(1.01 \pm 0.02)} \mu \mathrm{J} / \mathrm{g} \mathrm{K}$ between 0.1 and $8 \mathrm{~K}$. 
for the contributions of the empty apparatus and the Apiezon $\mathrm{N}$ grease.

An estimate of the contribution $C_{v}^{\text {TLS }}$ to the specific heat of the two-level systems of the glass has also been plotted as a function of temperature in Figs. 1 and 2 . This estimate has been obtained in the following manner. The total specific heat $C_{v}$ is assumed to consist of three terms:

$$
\begin{aligned}
C_{v} & =C_{v}^{\mathrm{TLS}}+C_{v}^{D}+C_{v}^{\mathrm{loc}} \\
& =a_{\mathrm{TLS}} T^{(1+\nu)}+\left(a_{D}+a_{\mathrm{exc}}\right) T^{3}+a_{\mathrm{loc}} T^{5} .
\end{aligned}
$$

The first term represents the contribution of the TLS and is assumed to be proportional to $T^{(1+\nu)}$, where the parameter $\nu$ is related to the energy dependence of the TLS density of states, $\rho=\rho_{0} E^{\nu}$, with $\nu=0-0.3$ [3]. The second term is a $T^{3}$-phonon contribution consisting of two parts: the Debye part, $a_{D} T^{3}$ related to the elastic constants, and an additional "excess" term characteristic for glasses, $a_{\text {exc }} T^{3}$, which is usually of the same order as the Debye term $[3,27]$. The third term proportional to $T^{5}$ only plays a role at higher temperatures and accounts for the broad maximum often observed in $C_{v} / T^{3}$ plots at about $5-10 \mathrm{~K}[1,2,28-30]$. In the literature this term is interpreted either as due to soft localized vibrations $[28,29]$ or to the onset of phonon dispersion [30]. All the terms related to $C_{v}$ will be discussed in more detail in a later publication.

In order to calculate $C_{v}^{\text {TLS }}$ the data of the total specific heat were fitted to Eq. (1) and, subsequently, the contributions of the $C_{v}^{D}$ and $C_{v}^{\text {loc }}$ terms were subtracted from the measured values of $C_{v}$. As illustrated for the $\mathrm{Pr}^{3+}$-doped glass in Fig. 3, $C_{v}^{\mathrm{TLS}} \propto T^{1.3}$ is the dominant term in Eq. (1) for $T<4 \mathrm{~K}$, whereas the second term proportional to $T^{3}$ becomes significant above this temperature and the third term $C_{v}^{\text {loc }} \propto T^{5}$ only at higher temperature $(T>8 \mathrm{~K})$. For the $\mathrm{Eu}^{3+}$ sample, where $C_{v}^{\text {TLS }} \propto T^{1.0}$ for $T<2 \mathrm{~K}$, the $T^{3}$ term is already important for $T>2 \mathrm{~K}$, and the $T^{5}$ term for $T>3 \mathrm{~K}$ (not shown). Thus, $C_{v} \approx C_{v}^{\mathrm{TLS}} \propto T^{1+\nu}$ for $T<2 \mathrm{~K}$ in both samples.

The values of $C_{v}^{\text {TLS }}$ for the $\mathrm{Pr}^{3+}$-doped sample shown in Fig. 1 (open triangles, right axis) follow a straight line, parallel to that of $\Gamma_{\text {hom }}$ versus $T$ for $T \leq 10 \mathrm{~K}$. The best fit of Eq. (1) between 0.2 and $15 \bar{K}$ yields $C_{v}^{\mathrm{TLS}}=a_{\mathrm{TLS}} T^{1+\nu}=(9.6 \pm 0.1) T^{(1.29 \pm 0.02)} \mu \mathrm{J} / \mathrm{gK}$, with $\nu=0.29 \pm 0.02$ (solid line). The $C_{v}^{\text {TLS }}$ values for the $\mathrm{Eu}^{3+}$-doped sample as a function of temperature between 0.1 and $5 \mathrm{~K}$ are shown in Fig. 2 (solid triangles, right scale). They are again parallel to those of $\Gamma_{\text {hom }}$ versus $T$, but now with a different slope. The best fit between 0.1 and $8 \mathrm{~K}$ is given by $C_{v}^{\mathrm{TLS}}=a_{\mathrm{TLS}} T^{1+\nu}=(3.5 \pm$ 0.1) $T^{(1.01 \pm 0.02)} \mu \mathrm{J} / \mathrm{g} \mathrm{K}$, with $\nu=0.01 \pm 0.02$ (solid line). Thus, $\Gamma_{\text {hom }}$ and $C_{v}^{\text {TLS }}$ both follow a $T^{\alpha}$ dependence with the same value of $\alpha$ for a given sample, but with different $\alpha$ 's for the two samples.

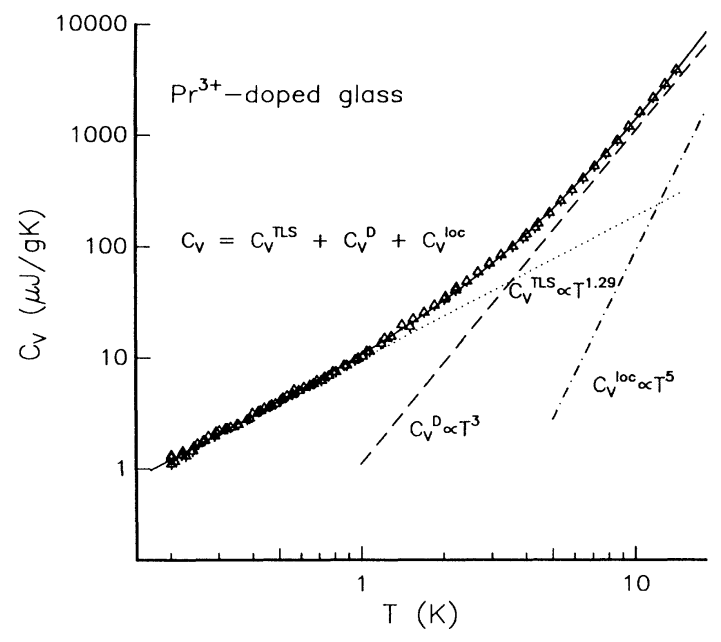

FIG. 3. Total specific heat, $C_{v}$, as a function of temperature for the $\mathrm{Pr}^{3+}$-doped silicate glass, between 0.2 and $15 \mathrm{~K}$. The data have been fitted with $C_{v}=C_{v}^{\mathrm{TLS}}+C_{v}^{D}+C_{v}^{\text {loc }}=(9.6 \pm 0.1) T^{1.29 \pm 0.02}+(1.11 \pm 0.05) T^{3}$ $+(0.0009 \pm 0.0003) T^{5} \mu \mathrm{J} / \mathrm{g} \mathrm{K}$. The data for the $\mathrm{Eu}^{3+}$-doped silicate glass, between 0.2 and $8 \mathrm{~K}$ (not shown), have been fitted with $C_{v}=(3.5 \pm 0.1) T^{1.01 \pm 0.02}+(1.18 \pm 0.05) T^{3}$ $+(0.035 \pm 0.003) T^{5} \mu \mathrm{J} / \mathrm{g} \mathrm{K}$. Typical errors in the $C_{v}$ measurements are about $5 \%$.

Theoretical models of dephasing in glasses assume that the optical transition is coupled to the TLS which, in turn, are coupled to the phonon bath [10,14,19-22]. "Flipping" of a TLS due to phonon interactions induces a change in the energy of the optical transition leading to dephasing. When assuming a TLS density of states proportional to $E^{\nu}$, these theories predict (i) a TLS contribution to the specific heat proportional to $T^{1+\nu}$, and (ii) a homogeneous linewidth due to TLS-induced dephasing proportional to $T^{\alpha}$ with $\alpha=(1+\nu) n / 3[10,14]$, or $\alpha=4+\nu-9 / n[20]$, depending on the treatment of the spatial averaging over the distribution of TLS. Since the value of $n$ depends on the type of coupling, we get $\alpha=1+\nu$ for $n=3$, i.e., for dipole-dipole coupling $[10,14]$. Therefore, both $\Gamma_{\text {hom }}$ and $C_{v}^{\text {TLS }}$ in a given sample should have the same temperature dependence. The results of Figs. 1 and 2 provide strong evidence for the consistency of these models if $\nu=0.3$ for the $\mathrm{Pr}^{3+}$-doped sample and $\nu=0.0$ for the $\mathrm{Eu}^{3+}$-doped sample.

We do not know yet whether the origin of the different values of $\nu$ is related to the composition of the silicate glass, which is different in the two samples or, less likely, to the rare-earth ion. We believe that a concentration of rare-earth ions of $0.1 \%$ to $0.25 \%$ would not contribute significantly to the number of two-level systems. On the other hand, the large difference in the $\mathrm{SiO}_{2}$ concentrations, $75 \%$ for the $\mathrm{Eu}^{3+}$ sample and $60 \%$ for the $\mathrm{Pr}^{3+}$ sample, might change the number of TLS considerably. Also the presence of glass modifiers, like $\mathrm{Na}_{2} \mathrm{O}, \mathrm{BaO}$, and 
$\mathrm{ZnO}$ in the $\mathrm{Eu}^{3+}$ sample, and $\mathrm{Li}_{2} \mathrm{O}, \mathrm{CaO}$, and $\mathrm{Al}_{2} \mathrm{O}_{3}$ in the $\mathrm{Pr}^{3+}$ sample, might change the nature of the TLS in the glass. Unfortunately, we were not able to obtain samples of $\mathrm{Eu}^{3+}$ and $\mathrm{Pr}^{3+}$ doped in identical silicate glasses. We are trying to procure such samples for future experiments. It is interesting, however, that, whatever the origin of the TLS is in the two samples, the same TLS can be held responsible for the optical dephasing and the temperature dependence of the specific heat within a given sample. It would otherwise be difficult to rationalize why the same exponent $\alpha$ appears in the temperature dependence of $\Gamma_{\text {hom }}$ and $C_{v}^{\text {TLS }}$.

We have further observed that the ratios of $\Gamma_{\text {hom }}$ to $C_{v}^{\text {TLS }}$ for the two samples are equal to within $20 \%$ to $30 \%$. This appears to indicate that the coupling strengths of the rare-earth ions to their respective glassy hosts are similar. The large difference in the absolute values of $\Gamma_{\text {hom }}$ in Figs. 1 and 2 then has to be attributed to the difference in the density of states of the TLS in the two samples.

In summary, experiments have been presented in which, for the first time, $\Gamma_{\text {hom }}$ and $C_{v}$ have been directly compared within one sample. The results are consistent with theoretical models that relate the specific heat and the optical dephasing through the two-level-system density of states as a function of energy under the assumption of a dipole-dipole coupling between the optical transition and the TLS.

We would like to thank R.M. Macfarlane for kindly providing us with the samples. Two of us (Th.S. and S.V.) enjoyed enlightening discussions with R. Silbey on the theory of two-level systems in glasses. Further we thank J. H. van der Waals for his constructive remarks regarding the presentation of the manuscript. The investigations were supported by the Netherlands Foundations for Physical Research (FOM) and Chemical Research (SON) with financial aid from the Netherlands Organization for Scientific Research (NWO).

* To whom correspondence should be addressed.

[1] W.A. Phillips, Rep. Prog. Phys. 50, 1657 (1987), and references therein; Amorphous Solids. Low-Temperature Properties, edited by W.A. Phillips, Topics in Current Physics 24 (Springer, Berlin, 1981), and references therein.

[2] R.C. Zeller and R.O. Pohl, Phys. Rev. B 4, 2029 (1971).

[3] J.C. Lasjaunias, A. Ravex, M. Vandorpe, and S. Hunklinger, Solid State Commun. 17, 1045 (1975).

[4] P.M. Selzer et al., Phys. Rev. Lett. 36, 813 (1976).
[5] J. Hegarty and W.M. Yen, Phys. Rev. Lett. 43, 1126 (1979)

[6] For a review, see Optical Linewidth in Glasses, edited by M.J. Weber and M.D. Sturge [J. Lumin. 36 (1987)].

[7] S. Völker, in Relaxation Processes in Molecular Excited States, edited by J. Fünfschilling (Kluwer, Dordrecht, 1989), p. 113, and references therein; Annu. Rev. Phys. Chem. 40, 499 (1989).

[8] L. Börjesson, A.K. Hassan, J. Swenson, and L.M. Torell, Phys. Rev. Lett. 70, 1275 (1993), and references therein.

[9] W.A. Phillips, J. Low Temp. Phys. 7, 315 (1972); P.W. Anderson, B.I. Halperin, and C.M. Varma, Philos. Mag. 25, 1 (1972).

[10] D.L. Huber, M.M. Broer, and B. Golding, Phys. Rev. Lett. 52, 2281 (1984).

[11] W.S. Brocklesby, B. Golding, and J.R. Simpson, Phys. Rev. Lett. 63, 1833 (1989).

[12] D.W. Pack, L.R. Narasimhan, and M.D. Fayer, J. Chem. Phys. 92, 4125 (1990).

[13] S. Uemura, K.M. Abedin, M. Okada, and H. Nakatsuka, J. Phys. Soc. Jpn. 60, 3557 (1991).

[14] M.M. Broer, B. Golding, W.H. Haemmerle, and J.R. Simpson, Phys. Rev. B 33, 4160 (1986); M.M. Broer and B. Golding, J. Opt. Soc. Am. B 3, 523 (1986).

[15] H.C. Meijers and D.A. Wiersma, Phys. Rev. Lett. 68, 381 (1992).

[16] W. Breinl, J. Friedrich, and D. Haarer, J. Chem. Phys. 81, 3915 (1984).

[17] K.A. Littau, M.A. Dugan, S. Chen, and M.D. Fayer, J. Chem. Phys. 96, 3484 (1992), and references therein.

[18] R. Wannemacher, J.M.A. Koedijk, and S. Völker, Chem. Phys. Lett. 206, 1 (1993).

[19] B. Jackson and R. Silbey, Chem. Phys. Lett. 171, 19 (1983).

[20] S.K. Lyo, Phys. Rev. Lett. 48, 688 (1982); S.K. Lyo and R. Orbach, Phys. Rev. B 29, 2300 (1984).

[21] P. Reineker and K. Kassner, in Optical Spectroscopy of Glasses, edited by I. Zschokke (Reidel, Dordrecht, 1986), p. 65 .

[22] R. Silbey and K. Kassner, J. Lumin. 36, 283 (1987), and references therein.

[23] Th. Schmidt et al. (unpublished).

[24] R.M. Macfarlane and R.M. Shelby, Opt. Commun. 45, 46 (1983)

[25] R. Bachman et al., Rev. Sci. Instrum. 43, 205 (1972).

[26] J. Baak et al., Physica (Amsterdam) 168C, 363 (1990).

[27] J. Zimmermann and G. Weber, Phys. Lett. 86A, 32 (1981).

[28] L. Gil, M.A. Ramos, A. Bringer, and U. Buchenau, Phys. Rev. Lett. 70, 182 (1993); U. Buchenau, Europhys. News 24, 77 (1993)

[29] U. Buchenau, Yu.M. Galperin, V.M. Gurevich, and H.R. Schober, Phys. Rev. B 43, 5039 (1991).

[30] H. von Löhneisen, H. Rüsing, and W. Sander, Z. Phys. B 60, 323 (1985). 